\title{
Interfacial Stresses in a Lap Shear Joint (LSJ): The "Transverse Groove Effect" (TGE)*
}

\author{
Ephraim SUHIR** and Tommi REINIKAINEN*** \\ ** Department of Electrical Engineering, University of California, Santa Cruz, CA; Department of \\ Mechanical Engineering, University of Maryland, College Park, MD; and ERS Co., Los Altos, CA; \\ 1156 High Street, Santa Cruz. 95064 \\ E-mail: suhire@aol.com \\ *** Nokia, Instituto Nokia de Tecnologia Manaus Brazil
}

\begin{abstract}
A simple and physically meaningful analytical model is developed for the evaluation of the interfacial stresses in a simple lap shear joint (LSJ). The emphasis is on the "peeling" stress, i.e., the stress acting in the through-thickness direction of the joint. The model is a modification and an extension of the model developed earlier for the shearing stress. Based on the developed model, we were able to explain a paradoxical situation due to the "transverse groove effect" (TGE). This effect has been detected by one of the authors of this paper (Reinikainen) from the finite-element-analysis (FEA) computations: deep enough transverse grooves deliberately introduced in the LSJ adherends ("pins") resulted in an appreciable reduction in the magnitude, and in a significant improvement in the uniformity, of the distribution of the interfacial shearing stress. It was determined that the stress relief due to the grooves was caused by the favorable effect of the increased interfacial compliance, while the adverse effect of the increased axial compliance of the pins (also due the grooves) was relatively small in a particular LSJ design and was suppressed by the favorable impact of the TGE. We show that the peeling stress in the LSJ is considerably lower than the interfacial shearing stress and that the TGE is even stronger for the peeling stress than for the shearing stress. This is because the grooves affect the peeling stress not only directly, through the increased interfacial compliance, but also indirectly, through the lower gradient of the interfacial shearing stress in the longitudinal direction. We show that this gradient can be reduced considerably if the interfacial compliance is increased, especially in a small size joint. We would like to point out that, although reasonably satisfactory agreement between the analytical and the finite-element analysis (FEA) predictions was obtained, the objective of our analysis was not so much to develop a more or less accurate analytical or a FEA model, but rather to demonstrate that the peeling stress, whatever technique is employed to evaluate it, can be reduced considerably by introducing the transverse grooves. The results of this article can be used in the analyses and design of LSJs, both in electronic packaging and beyond.
\end{abstract}

Key words: Shear Flow, Experimental Stress Analysis, Elasticity, Finite Element Method (FEM), System Analysis

\section{Introduction}

In this analysis we develop an analytical model for the evaluation of the interfacial stresses in a simple lap shear joint (LSJ). The emphasis is on the normal ("peeling") stress acting in the through-thickness direction. The model is a modification and an extension of the model 
developed earlier ${ }^{1}$ for the evaluation of the interfacial shearing stress. The earlier model explains the paradoxical situation initially detected on the basis of the finite element analysis (FEA): deep enough transverse grooves deliberately introduced in the adherends (pins) (Fig.1) can indeed reduce the magnitude and improve the uniformity of the distribution of the interfacial shearing stress in small size joints (Fig.2). Such a favorable "transverse groove effect" (TGE) takes place when the positive effect, in terms of the stress relief, due to the increase in the interfacial compliance in a particular LSJ "grooved" design suppresses the negative effect of the increase in the axial compliance of the pins. The objective of the analysis that follows is to find out to what extent the TGE could influence the peeling stress in a LSJ. We would like to point out that the objective of this analysis was not so much to develop a more or less accurate analytical or a FEA model, but rather to demonstrate that the peeling stress, whatever technique is used to evaluate it, can be reduced considerably by introducing the transverse grooves.

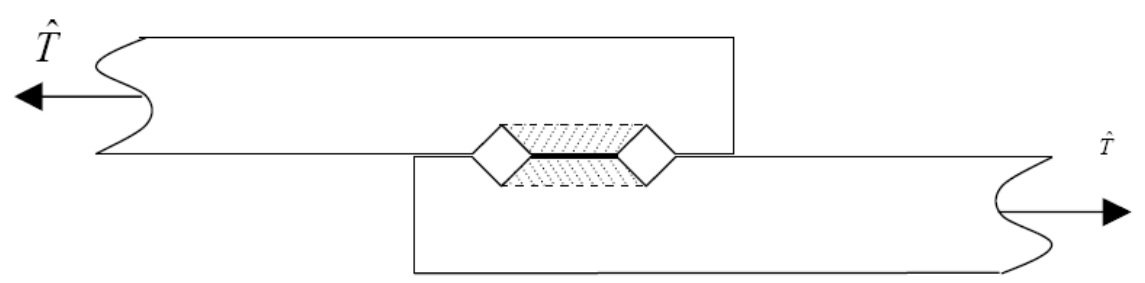

Figure 1 Deep enough transverse grooves deliberately introduced in the adherends (pins) can lead to an appreciatte reduction in the interfacial stresses

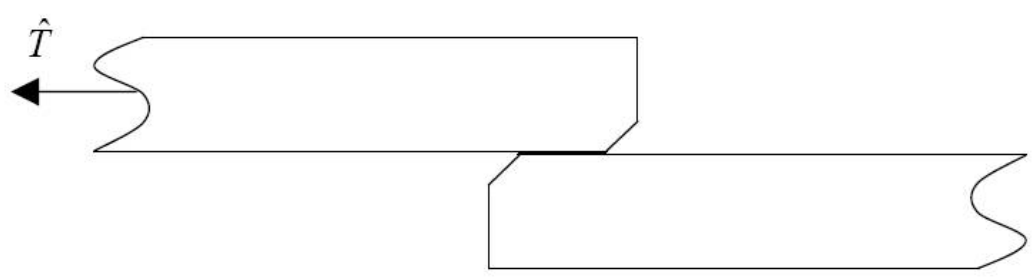

Figure 2 Conventional single lap shear joint

\section{Analysis}

\subsection{Basic Equation}

The forces, $T_{1}(x)$ and $T_{2}(x)$, acting in the cross-section $x$ of the adherends (pins) $\# 1$ and $\# 2$, respectively, of a simple lap shear joint (Fig.3) are related to the interfacial shearing stress, $\tau(x)$, by the formulas:

$$
T_{1}(x)=\hat{T}-\int_{-a}^{x} \tau(\xi) d \xi, T_{2}(x)=\int_{-a}^{x} \tau(\xi) d \xi
$$

where $\hat{T}$ are the applied tensile forces, and $a$ is half the joint's length. The origin of the coordinate $x$ is in the mid-cross-section of the joint. Clearly,

$$
\int_{-a}^{a} \tau(\xi) d \xi=\hat{T}
$$




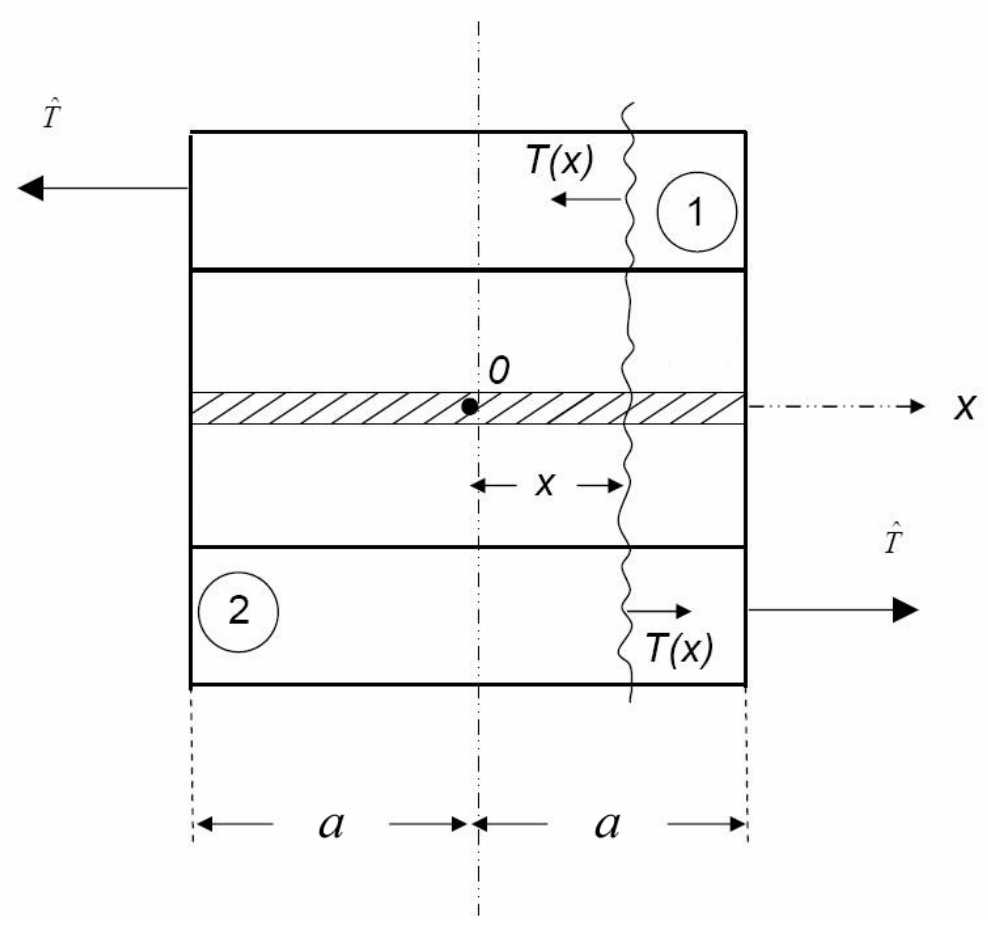

Figure 3 Forces acting in a LSJ with grooves

The formulas (1) and (2) satisfy the boundary conditions

$T_{1}(-a)=T_{2}(a)=\hat{T}, \quad T_{1}(a)=T_{2}(-a)=0$.

The equations of bending (equilibrium) of the pins can be written as

$$
\int_{-a-a}^{x} \int_{-a}^{x} p(\xi) d \xi d \xi_{1}=D w_{1}^{\prime \prime}(x)-\frac{h}{2} T_{1}(x)=-D w_{2}^{\prime \prime}(x)+\frac{h}{2} T_{2}(x)
$$

Here $p(x)$ is the interfacial peeling stress (i.e., the interfacial normal stress acting in the through-thickness direction of the joint), $h$ is the height of each pin,

$$
D=\frac{E h^{3}}{12}
$$

is its flexural rigidity, $E$ is Young's modulus of the pin material, $\quad w_{1}^{\prime \prime}(x)$ and $w_{2}^{\prime \prime}(x)$ are the curvatures of the pins due to their bending, and $w_{1}(x)$ and $w_{2}(x)$ are the pin deflection functions. The left part of the equations (4) is the bending moment due to the peeling stress $p(x)$. The terms in the right part containing the pin curvatures are the elastic bending moments acting in the pin cross-sections. The last terms in the right parts of the equations (4) are the bending moments due to the tensile forces $T_{1}(x)$ and $T_{2}(x)$. From (4) we find, by differentiation: 


$$
\int_{-a}^{x} p(\xi) d \xi=D w_{1}^{\prime \prime \prime}(x)+\frac{h}{2} \tau(x)=-D w_{2}^{\prime \prime \prime}(x)+\frac{h}{2} \tau(x) .
$$

The next differentiation yields:

$$
p(x)=D w_{1}{ }^{I V}(x)+\frac{h}{2} \tau^{\prime}(x)=-D w_{2}{ }^{I V}(x)+\frac{h}{2} \tau^{\prime}(x) .
$$

The peeling stress $p(x)$ must be self-equilibrated with respect to the bending moments, as well as with respect to the lateral forces, and therefore the following equilibrium conditions must be fulfilled:

$$
\int_{-a-a}^{a} \int^{x} p(\xi) d \xi d \xi_{1}=0, \quad \int_{-a}^{a} p(\xi) d \xi=0
$$

These conditions and the equations (4) and (6) lead to the following boundary conditions for the deflection functions $w_{1}(x)$ and $w_{2}(x)$ of the pins:

$$
w_{1}^{\prime \prime}(-a)=w_{2}^{\prime \prime}(a)=\frac{h \hat{T}}{2 D}, \quad w_{1}^{\prime \prime \prime}( \pm a)=w_{2}^{\prime \prime \prime}( \pm a)=-\frac{h}{2 D} \tau( \pm a) .
$$

It is natural to assume that the peeling stress $p(x)$ is related to the deflection functions

$$
\begin{aligned}
& w_{1}(x) \text { and } w_{2}(x) \text { as } \\
& p(x)=K\left[w_{2}(x)-w_{1}(x)\right],
\end{aligned}
$$

where $K$ is the interfacial spring constant with respect to the peeling loading. The formula (10) is based on an assumption that no peeling stresses can possibly occur, if the deflection functions $w_{1}(x)$ and $w_{2}(x)$ are the same at the given cross-section $x$. This formula reflects also an assumption that the peeling stress in the given cross-section is not affected by the states of stress and strain in the adjacent cross-sections. In an approximate analysis, the $K$ value can be evaluated by the formula:

$$
K=\frac{1}{2 \frac{1-v}{3 E} h+\frac{1-v_{0}}{E_{0}} h_{0}} .
$$

Here $E$ and $v$ are the elastic constants of the pin material, $E_{0}$ and $v_{0}$ are the elastic constants of the bonding material, and $h_{0}$ is the thickness of the bonding layer. The first term in the denominator of the formula (11) is the total through-thickness compliance of the pins. The structure of this term is based on Hooke's law. The factor " 3 " in front of the Young's modulus is included by analogy with the solution ${ }^{2}$ obtained for the interfacial compliance in shear. This solution considers that only the interfacial portions of the pins exhibit appreciable stresses, while their outer portions might be considerably understressed and do not contribute much to the physical behavior of the joint. The second term in the denominator in the formula (11) is the compliance of the bonding layer. This layer is fully 
involved in the state of stress at the interface.

Note that the boundary conditions (9) for the deflection functions $w_{1}(x)$ and $w_{2}(x)$ can be translated into the boundary conditions for the peeling stress function $p(x)$, using the formula (10), as follows:

$$
p^{\prime \prime}( \pm a)= \pm K \frac{h \hat{T}}{2 D}, \quad p^{\prime \prime \prime}( \pm a)=K \mu \tau( \pm a),
$$

where

$$
\mu=\frac{h}{D}=\frac{12}{E h^{2}}
$$

is the parameter of the joint's finite flexural rigidity. In the case of very stiff ("high") pins and/or pins made of a high-modulus materials, this parameter is small.

Differentiating the formula (10) four times with respect to the coordinate $x$, we obtain:

$$
p^{I V}(x)=K\left[w_{2}{ }^{I V}(x)-w_{1}^{I V}(x)\right]
$$

Solving the equations (7) for the fourth derivatives of the deflection functions and introducing the obtained expressions into the formula (14) we obtain the following equation for the peeling stress function $p(x)$ :

$$
p^{I V}(x)+4 \beta^{4} p(x)=K \mu \tau^{\prime}(x)
$$

where

$$
\beta=\sqrt[4]{\frac{K}{2 D}}
$$

is the parameter of the interfacial peeling stress.

The longitudinal interfacial displacements of the pins can be sought, in an approximate analysis, in the form:

$$
\begin{aligned}
& u_{1}(x)=\lambda_{1} \int_{-a}^{x} T_{1}(\xi) d \xi+\kappa_{1} \tau(x)+\frac{h}{2} w_{1}^{\prime}(x), \\
& u_{2}(x)=\lambda_{2} \int_{-a}^{x} T_{2}(\xi) d \xi-\kappa_{2} \tau(x)+\frac{h}{2} w_{2}^{\prime}(x),
\end{aligned}
$$

where $\lambda_{1}$ are the axial compliances of the pins, and $\kappa_{1}$ are their interfacial compliances.

The formulas for these compliances are obtained and discussed in detail in the Ref.1.

The condition of the compatibility of the displacements (17) can be written as:

$$
u_{1}(x)=u_{2}(x)-\kappa_{0} \tau(x),
$$

where $\kappa_{0}$ is the interfacial compliance of the bonding layer. Introducing the formulas (17) into the condition (18) and differentiating the obtained expression with respect to the coordinate $x$, we obtain the following equation for the shearing stress function $\tau(x)$ : 


$$
\tau^{\prime}(x)-k^{2} \int_{-a}^{x} \tau(\xi) d \xi=-\mu \int_{-a-a}^{x} \int_{-a}^{x} p(\xi) d \xi d \xi_{1}-\left(\lambda_{1}+\frac{h^{2}}{4 D}\right) \hat{T}
$$

where

$$
k=\sqrt{\frac{\lambda}{\kappa}}
$$

is the parameter of the interfacial shearing stress,

$$
\lambda=2 \lambda_{1}+\frac{h^{2}}{2 D}
$$

is the total axial compliance of the joint, with consideration of its finite flexural rigidity, and

$$
\kappa=\kappa_{0}+2 \kappa_{1}
$$

is the total interfacial compliance of the joint.

From (19), considering the formula (2) and the first equilibrium condition in (8), we obtain the following boundary condition for the shearing stress function $\tau(x)$;

$$
\tau^{\prime}( \pm a)= \pm\left(\lambda_{1}+\frac{h^{2}}{4 D}\right) \frac{\hat{T}}{\kappa}=\frac{\lambda \hat{T}}{2 \kappa} .
$$

From (19) we find, by differentiation:

$$
\tau^{\prime \prime}(x)-k^{2} \tau(x)=-\frac{\mu}{\kappa} \int_{-a}^{x} p(\xi) d \xi .
$$

In an approximate analysis, we assume that the flexural rigidity, $D$, of each pin is significant and the $\mu$ value is small enough, so that the right part in the equation (24) can be put equal to zero. With such a simplifying assumption the interfacial shearing stress function $\tau(x)$ can be determined from the homogeneous equation

$$
\tau^{\prime \prime}(x)-k^{2} \tau(x)=0 \text {. }
$$

By putting the right part in (24) equal to zero, we, actually, assume that the shearing stress is not affected by the peeling stress. This assumption was first used in Ref.3 in application to a thin film structure subjected to the change in temperature. The accuracy (acceptability) of such an assumption was then confirmed in Ref.4 for bi-material assemblies with comparable thicknesses of the adherends. In the current analysis we assume that this assumption is valid also for assemblies (joints) subjected to mechanical loading. Note that the equation (25) is always fulfilled, as one could see from (24), at the joint's end $x=a$. The equation (25) has the following solution that satisfies the boundary condition (23):

$$
\tau(x)=\frac{\lambda \hat{T}}{2 k \kappa} \frac{\cosh k x}{\sinh k a}=\frac{k \hat{T}}{2} \frac{\cosh k x}{\sinh k a} .
$$

Although this solution coincides with the solution obtained in Ref.1, the parameter $k$ of the interfacial shearing stress in (26) is obtained on the basis of the formula (21) for the 
axial compliance, which is determined, unlike in Ref.1, with consideration of the finite flexural rigidity $D$. This circumstance, if this rigidity is not very large, results in a somewhat larger axial compliance of the pins and, hence, in a larger value of the parameter $k$ of the interfacial shearing stress and the interfacial shearing stress itself.

Considering the solution (26), the second boundary condition in (12) yields:

$$
p^{\prime \prime \prime}( \pm a)=K \mu \frac{k \hat{T}}{2} \operatorname{coth} k a=K k h \frac{\hat{T}}{2 D} \operatorname{coth} k a .
$$

Thus, the interfacial peeling stress function $p(x)$ should satisfy the first boundary condition in (12) and the condition (27). The basic equation for this stress can be obtained from (15) and (26) as follows:

$$
p^{I V}(x)+4 \beta^{4} p(x)=\beta^{4} k^{2} \hat{T} h \frac{\sinh k x}{\sinh k a} .
$$

\subsection{Peeling Stress}

While the interfacial shearing stress is symmetric with respect to the mid-cross section of the joint, the interfacial peeling stress is anti-symmetric with respect to this cross-section. We seek the solution to the equation (28) therefore in the form:

$p(x)=C_{1} V_{1}(\beta x)+C_{3} V_{3}(\beta x)+p_{0} \frac{\sinh k x}{\sinh k a}$,

where the functions $V_{i}(\beta x), i=0,1,2,3$, are expressed as follows (see, for instance, ${ }^{5}$ ):

$V_{0}(\beta x)=\cosh \beta x \cos \beta x, \quad V_{0}(\beta x)=\sinh \beta x \sin \beta x$,

$V_{1,3}(\beta x)=\frac{1}{\sqrt{2}}(\cosh \beta x \sin \beta x \pm \sinh \beta x \cos \beta x)$,

and obey the following simple rules of differentiation:

$$
\begin{aligned}
& V_{0}^{\prime}(\beta x)=-\beta \sqrt{2} V_{3}(\beta x), V_{1}^{\prime}(\beta x)=\beta \sqrt{2} V_{0}(\beta x), \\
& V_{2}^{\prime}(\beta x)=\beta \sqrt{2} V_{1}(\beta x), V_{3}^{\prime}(\beta x)=\beta \sqrt{2} V_{2}(\beta x) .
\end{aligned}
$$

In the equation (29),

$$
p_{0}=\frac{\varsigma^{4}}{1+\varsigma^{4}} \frac{\hat{T} h k^{2}}{4},
$$

and

$$
\varsigma=\frac{\beta \sqrt{2}}{k}
$$

is the ratio that considers the relative level of the parameters of the interfacial peeling stress and the interfacial peeling stress. The first two terms in (29) is the general solution to the homogeneous equation that corresponds to the inhomogeneous equation (28). The last term in (29) is the particular solution to the inhomogeneous equation (28).

In order to determine the constants $C_{1}$ and $C_{2}$ of integration in the solution (29) one can 
either use the first boundary condition in (12) and the condition (27) or to use directly the conditions (8) of equilibrium. Employing the second option, we obtain the following equations for the constants of integration:

$-V_{2}(u) C_{1}-\left[1-V_{0}(u)\right] C_{3}=p_{0} \varsigma \tanh \frac{k a}{2}, \quad V_{3}(u) C_{1}-V_{1}(u) C_{3}=-p_{0} \varsigma^{2}$,

where

$$
u=\beta a=a \sqrt[4]{\frac{K}{2 D}}
$$

is the dimensionless parameter of the interfacial peeling stress. The equations (34) yield:

$$
\begin{aligned}
& C_{1}=-p_{0} \varsigma \frac{\varsigma\left[1-V_{0}(u)\right]+V_{1}(u) \tanh \frac{k a}{2}}{V_{1}(u) V_{2}(u)+V_{3}(u)\left[1-V_{0}(u)\right]}= \\
& =-p_{0} \varsigma \frac{\varsigma \sqrt{2}(1-\cosh u \cos u)+(\cosh u \sin u+\sinh u \cos u) \tanh \frac{k a}{2}}{(\cosh u-\cos u)(\sinh u+\sin u)}, \\
& C_{3}=p_{0} \varsigma \frac{\varsigma V_{2}(u)-V_{3}(u) \tanh \frac{k a}{2}}{V_{1}(u) V_{2}(u)+V_{3}(u)\left[1-V_{0}(u)\right]}= \\
& =p_{0} \varsigma \frac{\varsigma \sqrt{2} \sinh u \sin u-(\cosh u \sin u-\sinh u \cos u) \tanh \frac{k a}{2}}{(\cosh u-\cos u)(\sinh u+\sin u)}
\end{aligned}
$$

The solution (29) leads to the following expression for the peeling stress:

$$
\begin{aligned}
& p(x)=\frac{p_{0} \varsigma}{\sqrt{2}} \frac{\varsigma \sqrt{2} \sinh u \sin u-(\cosh u \sin u-\sinh u \cos u) \tanh \frac{k a}{2}}{(\cosh u-\cos u)(\sinh u+\sin u)}(\cosh \beta x \sin \beta x-\sinh \beta x \cos \beta x)- \\
& -\frac{p_{0} \varsigma}{\sqrt{2}} \frac{\varsigma \sqrt{2}(1-\cosh u \cos u)+(\cosh u \sin u+\sinh u \cos u) \tanh \frac{k a}{2}}{(\cosh u-\cos u)(\sinh u+\sin u)}(\cosh \beta x \sin \beta x+\sinh \beta x \cos \beta x)+ \\
& +p_{0} \frac{\sinh k x}{\sinh k a} .
\end{aligned}
$$

\subsection{Long Joint}

In the special case of a long (large $a$ values) joint and/or a joint with a stiff interface (large $\beta$ and $k$ values) the solution (37) can be simplified as follows:

$$
p(x)=\frac{p_{0}}{2} \varsigma e^{-\beta(a-x)}[(\varsigma-\sqrt{2}) \cos (\beta(a-x))-\varsigma \sin (\beta(a-x))]+p_{0} e^{-k(a-x)}
$$

Such an approximation can be used when $u \geq 2.5$ and $k a \geq 6$. Putting the origin at the joint's end we obtain the expression for the peeling stress in a long joint in the following simple form: 
$p(x)=\frac{p_{0}}{2} \varsigma e^{-\beta x}[(\varsigma-\sqrt{2}) \cos \beta x-\varsigma \sin \beta x]+p_{0} e^{-k x}$.

At the joint's end

$$
p=p_{\max }=p_{0}\left[\frac{\varsigma(\varsigma-\sqrt{2})}{2}+1\right]=\frac{\hat{T} h k^{2}}{4} \chi(\varsigma), .
$$

where the factor

$$
\chi(\varsigma)=\frac{\varsigma^{4}}{1+\varsigma^{4}}\left[\frac{\varsigma(\varsigma-\sqrt{2})}{2}+1\right]
$$

considers the effect of the parameter $\beta$ of the peeling stress on the maximum value of this stress in a long and/or stiff joint. This factor changes from zero to infinity, when the parameter $\beta$ (and the ratio $\varsigma$ ) changes from zero to infinity. For large enough $\beta$ values the maximum peeling stress at the end of a long enough joint is independent of the parameter $k$ of the interfacial shearing stress and can be evaluated as

$$
p_{\max }=\hat{T h}\left(\frac{\beta}{2}\right)^{2}
$$

As evident from the formula (40), the maximum value of the peeling stress in a long and/or stiff enough joint is joint length independent.

The formula (26) yields:

$$
\tau(x)=\frac{k \hat{T}}{2} e^{-k(a-x)} .
$$

Hence, the shearing stress concentrates in this case at the joint's end, and its maximum value

$$
\tau=\tau_{\max }=\frac{k \hat{T}}{2}
$$

is independent of the joint's length.

\subsection{Short Joint}

In another special case of a short (small $a$ values) joint the solution (37) yields:

$$
p(x)=p_{0} \varsigma^{4}\left(\frac{k a}{2}\right)^{2} \frac{x^{3}}{a^{3}}=\frac{\varsigma^{6}}{1+\varsigma^{4}} \frac{\hat{T} h k^{2}}{4}\left(\frac{k a}{2}\right)^{2} \frac{x^{3}}{a^{3}}
$$

Such an approximation can be used when $u \leq 0.2, k a \leq 0.4$. The maximum value of the peeling stress in this case is

$$
p_{\max }=\frac{\varsigma^{6}}{1+\varsigma^{4}} \frac{\hat{T} h k^{2}}{4}\left(\frac{k a}{2}\right)^{2}
$$

and is strongly affected by the joint's size. The peeling stress is very low in small size joints. The interfacial shearing stress is expressed for a short and/or compliant joint by the formula 


$$
\tau(x)=\frac{\hat{T}}{2 a}\left[1+\frac{(k x)^{2}}{2}\right]
$$

that can be obtained from the formula (26). Hence, the shearing stress is distributed over the joint's length in accordance with a parabolic law.

From (42) and (43) we conclude that in a short joint the ratio of the maximum peeling stress to the maximum shearing stress is

$$
\frac{p_{\max }}{\tau_{\max }}=2 \frac{\varsigma^{6}}{1+\varsigma^{4}} \frac{h}{a}\left(\frac{k a}{2}\right)^{4} .
$$

This formula indicates that the maximum peeling stress in a small size joint could be considerably lower than the maximum interfacial shearing stress.

\section{Numerical Examples}

\subsection{Interfacial Shearing Stress}

Consider first a conventional, "ungrooved", lap shear joint with the following Input data (Ref.1):

- Joint's length $2 a=2 \mathrm{~mm}$,

- External force $100 \mathrm{~N}=10.204 \mathrm{kgf}$,

- Joint'width $b=1.5 \mathrm{~mm}$,

- External force per unit width $\hat{T}=6.803 \mathrm{kgf} / \mathrm{mm}$,

- Thickness (height) of the adherends ("pins") $h=3 \mathrm{~mm}$,

- Elastic constants of the adherend material: $E=210 \mathrm{GPa}=21428 \mathrm{kgf} / \mathrm{mm}^{2}, v=0.3$,

- Thickness of the adhesive (solder): $\mathrm{h}_{0}=0.1 \mathrm{~mm}$,

- Elastic constants of the adhesive (solder) material: $E=44.3 \mathrm{GPa}=4520 \mathrm{kgf} / \mathrm{mm}^{2}$, $v=0.35$.

Calculated Data:

- The total axial compliance of the joint

$$
\lambda=2 \frac{1-v}{E h}=2 \frac{1-0.3}{21428 \times 3.0}=2.1778 \times 10^{-5} \mathrm{~mm} / \mathrm{kg}
$$

- Shear moduli of the materials of the pins and of the adhesive (solder):

$$
\begin{aligned}
& G=\frac{E}{2(1+v)}=\frac{21428}{2 \times 1.3}=8241.5 \mathrm{~kg} / \mathrm{mm}^{2} \\
& G_{0}=\frac{E_{0}}{2\left(1+v_{0}\right)}=\frac{4520}{2 \times 1.35}=1674.1 \mathrm{~kg} / \mathrm{mm}^{2}
\end{aligned}
$$

- Thickness (height) to length ratio for pins: $\frac{h}{a}=\frac{3.0}{1.0}=3 \geq 0.2$

- Thickness (height) to length ratio for solder: $\frac{h}{a}=\frac{0.1}{1.0}=0.1 \leq 0.5$

- Interfacial compliance of each pin 


$$
\kappa_{1}=\frac{3-v}{\pi} \frac{2 a}{G}=\frac{2.7}{\pi} \frac{2 \times 1.0}{8241.5}=20.8563 \times 10^{-5} \mathrm{~mm}^{3} / \mathrm{kgf}
$$

- Interfacial compliance of the solder

$$
\kappa_{0}=\frac{h_{0}}{G_{0}}=\frac{0.1}{1674.1}=5.9734 \times 10^{-5} \mathrm{~mm}^{3} / \mathrm{kgf}
$$

- The total interfacial compliance

$\kappa=2 \kappa_{1}+\kappa_{0}=2 \times 20.8563 \times 10^{-5}+5.9734 \times 10^{-5}=47.6860 \times 10^{-5} \mathrm{~mm}^{3} / \mathrm{kgf}$

- Parameter of the interfacial compliance

$$
k=\sqrt{\frac{2 \lambda}{\kappa}}=\sqrt{\frac{2 \times 2.1778 \times 10^{-5}}{47.6860 \times 10^{-5}}}=0.3022 \mathrm{~mm}^{-1}
$$

- The formulas (9) result in the following values of the interfacial shearing stress at the joint ends and in its mid-cross-section:

$\tau_{0}( \pm a)=\frac{k \hat{T}}{2} \operatorname{coth} k a=\frac{0.3022 \times 6.803}{2} \operatorname{coth}(0.3022 \times 1.0)=3.5044 \mathrm{~kg} f / \mathrm{mm}^{2}=$ $=34.343 \mathrm{MPa}$

$$
\begin{aligned}
\tau_{0}(0)=\frac{k \hat{T}}{2} & \frac{1}{\sinh k a}=\frac{0.3022 \times 6.803}{2} \frac{1}{\sinh (0.3022 \times 1.0)}=3.3504 \mathrm{kgf} / \mathrm{mm}^{2}= \\
= & 32.834 \mathrm{MPa}
\end{aligned}
$$

Let us evaluate now the shearing stress with consideration of the effect of the finite flexural rigidity of the pins on the axial compliance.

- The axial compliance of one pin with consideration of its finite flexural rigidity

$\lambda=\frac{1-v}{E h}+\frac{h^{2}}{4 D}=\frac{1-v}{E h}+\frac{3}{E h}=\frac{1-0.3}{21428 \times 3.0}+\frac{3}{21428 \times 3.0}=5.7557 \times 10^{-5} \mathrm{~mm} / \mathrm{kg}$

- Factor of the interfacial shearing stress with consideration of the finite flexural rigidity of the pins

$k=\sqrt{\frac{2 \lambda}{\kappa}}=\sqrt{\frac{2 \times 5.7557 \times 10^{-5}}{47.6860 \times 10^{-5}}}=0.4913 \mathrm{~mm}^{-1}$

- The interfacial shearing stress at the joint ends and in its mid-cross-section:

$\tau_{0}( \pm a)=\frac{k \hat{T}}{2} \operatorname{coth} k a=\frac{0.4913 \times 6.803}{2} \operatorname{coth}(0.4913 \times 1.0)=3.6708 \mathrm{kgf} / \mathrm{mm}^{2}=36.0 \mathrm{MPa}$

$\tau_{0}(0)=\frac{k \hat{T}}{2} \frac{1}{\sinh k a}=\frac{0.4913 \times 6.803}{2} \frac{1}{\sinh (0.4913 \times 1.0)}=3.2685 \mathrm{kgf} / \mathrm{mm}^{2}=32.0 \mathrm{MPa}$

Thus, consideration of the finite flexural rigidity led, for a regular ("ungrooved") joint to about $4.75 \%$ increase in the maximum shearing stress at the joint ends and to about $2.50 \%$ decrease in the minimum shearing stress in the mid-cross-section, i.e., to a less uniform distribution of the interfacial shearing stress. The finite element analysis (FEA) mesh is 
shown in Fig. 4 and the FEA data are $\tau_{0}( \pm a)=46.0 \mathrm{MPa}$ and $\tau_{0}(0)=27.0 \mathrm{MPa}$ at the assembly edges and at its mid-cross-section, respectively. We attribute the discrepancy between the analytical and the FEA predictions to the approximate analytical evaluations as well as to the well-known fact that the FEA usually leads to a singularity (infinitely high stresses) at the dissimilar material interfaces. This effect is expected to be especially strong in small size assemblies, when the effect of the stress fields at the edges is particularly strong. We would like to point out, however, that our objective is not to come up with a highly accurate analytical or FEA predictions, but rather to demonstrate the stress relieving role of the transverse grooves.

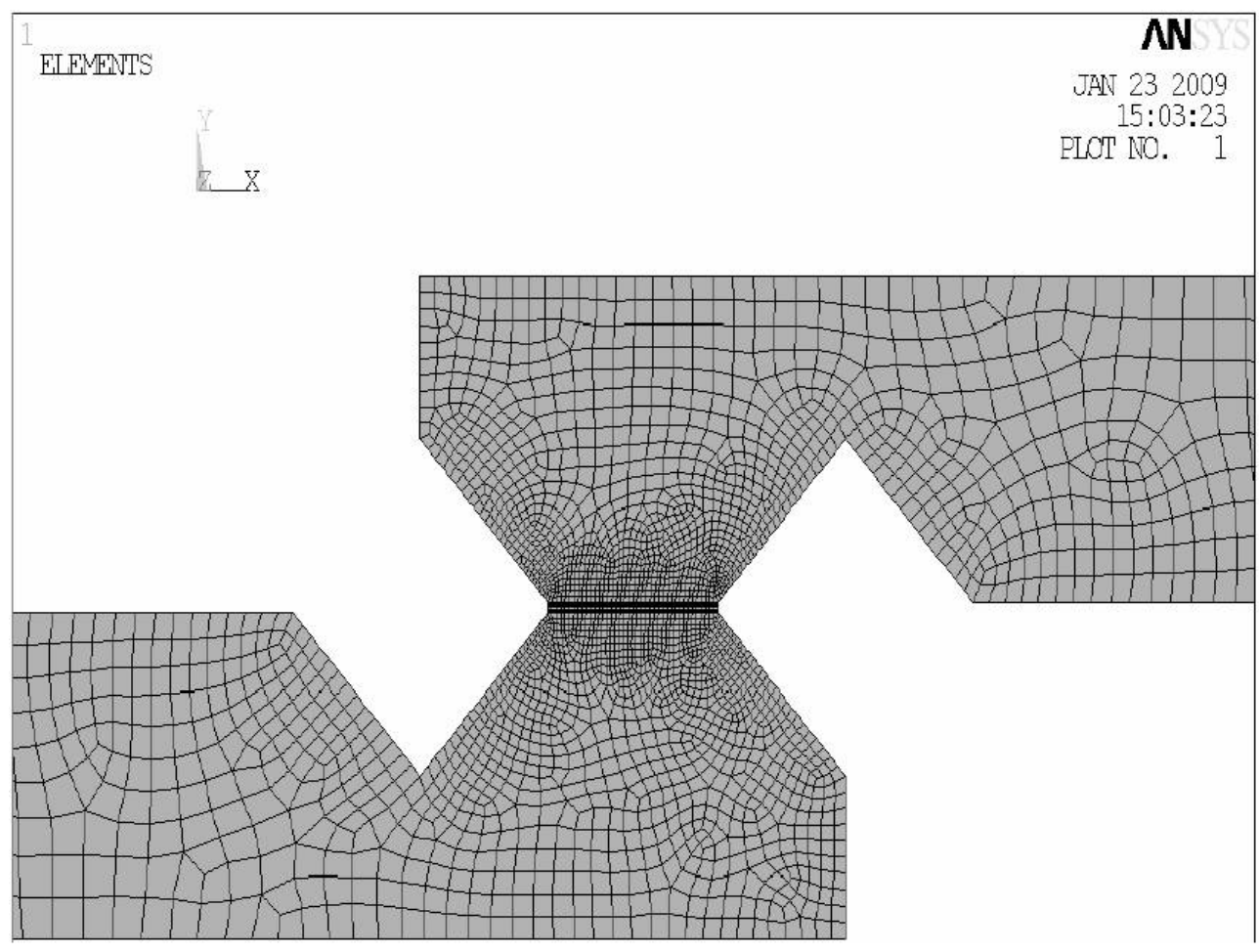

Figure4 Finite element simulation for grooved-lap test for solder joints

2. Examine now the case when $1.5 \mathrm{~mm}$ deep transverse groves are made in the joint's pins. The pins have become, because of that, two times thinner and the axial compliance of the joints has become twice as large: $\lambda=4.3556 \times 10^{-5} \mathrm{~mm} / \mathrm{kg}$. The thickness (height) to the half the joint's length ratio of the portions of the pins that became parts of the bonding structure is $3.00 \mathrm{~mm} / 1.0 \mathrm{~mm}=3.0$, so that the formula $(15)$ should be used to evaluate their interfacial compliance. This yields:

$$
\kappa=3 \frac{3-v}{\pi} \frac{2 a}{G}=3 \frac{2.7}{\pi} \frac{2 \times 1.0}{8241.5}=62.569 \times 10^{-5} \mathrm{~mm}^{3} / \mathrm{kgf}
$$

The interfacial compliance of the outer portions of the pins is three times lower: $\kappa=20.8563 \mathrm{~mm}^{3} / \mathrm{kg} f$. The interfacial compliance of the adhesive (solder) has not change: $\kappa_{0}=5.9734 \times 10^{-5} \mathrm{~mm}^{3} / \mathrm{kgf}$ The total interfacial compliance of the 
"grooved" joint is

$\kappa=2 \times 20.8563 \times 10^{-5}+2 \times 62.5690 \times 10^{-5}+5.9734 \times 10^{-5}=172.8240 \times 10^{-5} \mathrm{~mm}^{3} / \mathrm{kgf}$

The parameter of the interfacial stress in a joint with grooves is

$$
k=\sqrt{\frac{4.3556 \times 10^{-5}}{172.8240 \times 10^{-5}}}=0.1588 \mathrm{~mm}^{-1}
$$

Thus, in the grooved joint the parameter of the interfacial shearing stress is approximately half of the value of this parameter in the conventional lap shear joint. The formulas (9) yield:

$$
\begin{aligned}
& \tau_{0}( \pm a)=\frac{k \hat{T}}{2} \operatorname{coth} k a=\frac{0.1588 \times 6.803}{2} \operatorname{coth}(0.1588 \times 1.0)=3.4301 \mathrm{kgf} / \mathrm{mm}^{2}=33.6 \mathrm{MPa} \\
& \tau_{0}(0)=\frac{k \hat{T}}{2} \frac{1}{\sinh k a}=\frac{0.1588 \times 6.803}{2} \frac{1}{\sinh (0.1588 \times 1.0)}=3.3873 \mathrm{kgf} / \mathrm{mm}^{2}=33.2 \mathrm{MPa}
\end{aligned}
$$

Note that in an ideal case of uniformly distributed interfacial shearing stresses these stresses would be

$$
\tau_{0}=\frac{\hat{T}}{2 a}=\frac{6.803}{2 \times 1.0}=3.4015 \mathrm{~kg} / \mathrm{mm}^{2}=33.330 \mathrm{MPa}
$$

If the finite flexural rigidity of the pins is considered, then the axial compliance of each pin would be

$$
\lambda=4.3556 \times 10^{-5}+\frac{3}{21428 \times 1.5}=13.6892 \times 10^{-5} \mathrm{~mm} / \mathrm{kgf}
$$

and the parameter of the interfacial shearing stress would be

$$
k=\sqrt{\frac{13.6892 \times 10^{-5}}{172.8240 \times 10^{-5}}}=0.2814 \mathrm{~mm}^{-1}
$$

The interfacial shearing stresses are

$$
\tau_{0}( \pm a)=\frac{k \hat{T}}{2} \operatorname{coth} k a=\frac{0.2814 x 6.803}{2} \operatorname{coth}(0.2814 x 1.0)=3.4908 \mathrm{kgf} / \mathrm{mm}^{2}=35.6 \mathrm{MPa}
$$

The FEA result is

$$
\tau_{0}(0)=\frac{k \hat{T}}{2} \frac{1}{\sinh k a}=\frac{0.2814 x 6.803}{2} \frac{1}{\sinh (0.2814 x 1.0)}=3.3569 \mathrm{kgf} / \mathrm{mm}^{2}=32.9 \mathrm{MPa}
$$

Thus, consideration of the finite flexural rigidity led for a "grooved" joint to about $1.77 \%$ increase in the maximum shearing stress at the joint ends and to about $0.90 \%$ decrease in the minimum shearing stress in the mid-cross-section, i.e., to a less uniform distribution of the interfacial shearing stress. The effect of the finite flexural rigidity of the pins is much less in the case of "grooved" joints than in the case of conventional, "ungrooved", joints.

The FEA predictions are $\tau_{0}( \pm a)=38.0 \mathrm{MPa}$ and $\tau_{0}(0)=32.5 \mathrm{MPa}$.

\subsection{Interfacial Peeling Stress}

Consider first a conventional, "ungrooved", lap shear joint, with the same input data as in Example \#1. 
- The interfacial spring constant of the joint in the through-thickness direction

$K=\frac{1}{2 \frac{1-v}{3 E} h+\frac{1-v_{0}}{E_{0}} h_{0}}=\frac{1}{2 \frac{0.7}{3 \times 21428} 3.0+\frac{0.65}{4520} 0.1}=12544.5 \mathrm{~kg} / \mathrm{mm}^{3}$

- Flexural rigidity of a pin

$$
D=\frac{E h^{3}}{12}=\frac{21428 \times 27}{12}=48213 \mathrm{kgfxm}
$$

- $\quad$ Parameter of the interfacial peeling stress

$$
\beta=\sqrt[4]{\frac{K}{2 D}}=\sqrt[4]{\frac{12544.5}{2 \times 48213}}=0.6006 \mathrm{~mm}^{-1}
$$

- Interfacial parameters ratio

$$
\varsigma=\frac{\beta \sqrt{2}}{k}=\frac{0.6006 \sqrt{2}}{0.4913}=1.7288
$$

- Dimensionless parameters of the interfacial shearing and interfacial peeling stresses

$$
k a=0.4913 \times 1.0=0.4913 ; u=\beta a=0.6006 x 1.0=0.6006
$$

- Peeling stress at the end of a short joint

$p_{0}=\frac{\varsigma^{4}}{1+\varsigma^{4}} \frac{\hat{T} h k^{2}}{4}=\frac{1.7288^{4}}{1+1.7288^{4}} \frac{6.803 \times 3 \times 0.4913^{2}}{4}=1.1076 \mathrm{~kg} / \mathrm{mm}^{2}=10.8 \mathrm{MPa}$

- The maximum peeling stress in a long joint is much higher:

$p_{\max }=p_{0}\left[\frac{\varsigma(\varsigma-\sqrt{2})}{2}+1\right]=\frac{\hat{T h} k^{2}}{4} \chi(\varsigma)=\frac{6.803 \times 3.0 \times 0.4913^{2}}{4} \times 1.1439=1.4087 \mathrm{kgf} / \mathrm{mm}^{2}=$ $=13.8 \mathrm{MPa}$,

but even this stress is lower than the shearing stress in a short joint. The FEA prediction is $p_{\max }=12.0 \mathrm{MPa}$

4. In the case of a grooved LSJ, the parameter of the interfacial shearing stress is $k=0.2814 \mathrm{~mm}^{-1}$. Then the peeling/shearing parameter ratio is $\varsigma=\frac{\beta \sqrt{2}}{k}=\frac{0.6006 \sqrt{2}}{0.2814}=3.0184$.

Using the approximation of a small size LSJ, we have:

$p_{0}=\frac{\varsigma^{4}}{1+\varsigma^{4}} \frac{\hat{T} h k^{2}}{4}=\frac{3.0184^{4}}{1+3.0184^{4}} \frac{6.803 \times 1.5 \times 0.2814^{2}}{4}=0.200 \mathrm{kgf} / \mathrm{mm}^{2}=1.96 \mathrm{MPa}$

The maximum peeling stress in a long joint is

$p_{\max }=p_{0}\left[\frac{\varsigma(\varsigma-\sqrt{2})}{2}+1\right]=\frac{\hat{T h} k^{2}}{4} \chi(\varsigma)=\frac{6.803 \times 1.5 \times 0.2814^{2}}{4} \times 3.387=0.6774 \mathrm{kgf} / \mathrm{mm}^{2}=$

$=6.6 M P a$,

The FEA prediction is $p_{\max }=7.5 \mathrm{MPa}$. 


\section{Conclusions}

- Simple analytical stress models are developed for the evaluation of the interfacial shearing and peeling stresses in lap shear joints. Consideration of the finite flexural rigidity of the joints results in an appreciable increase in the predicted interfacial shearing stress. In the carried out example, consideration of the finite flexural rigidity of the adherends (pins) led, for a regular ("ungrooved") joint, to about $4.75 \%$ increase in the maximum shearing stress at the joint ends and to about $2.50 \%$ decrease in the minimum shearing stress in its mid-cross-section, i.e., to a less uniform distribution of the interfacial shearing stress. For a "grooved" joint, consideration of the finite flexural rigidity led to about $1.77 \%$ increase in the maximum shearing stress at the joint ends and to about $0.90 \%$ decrease in the minimum shearing stress in the mid-cross-section. Thus, the effect of the finite flexural rigidity of the pins is much less in the case of "grooved" joints than in the case of conventional joints.

- The peeling stresses in lap shear joints are significantly lower than the interfacial shearing stresses. In the carried out example, the maximum shearing stress in the ungrooved joint with consideration of the effect of the flexural rigidity is $3.6708 \mathrm{kgf} / \mathrm{mm}$ sq., while the maximum peeling stress is only $0.2155 \mathrm{kgf} / \mathrm{mm}$ sq. Even in a long joint the maximum peeling stress is only $1.4087 \mathrm{kgf} / \mathrm{mm}$.

- The "transverse groove effect" (TGE) is more strongly pronounced for the peeling stress than for the shearing stress. In the carried out example the maximum peeling stress in a small size joint is only $0.036 \mathrm{kgf} / \mathrm{mm}$.sq. , i.e., only about $17 \%$ of the peeling stress level in an ungrooved LSJ.

\section{References}

1. E.Suhir, T. Reinikainen, "On a Paradoxical Situation Related to Lap Shear Joints: Could Transverse Grooves in the Adherends Reduce the Interfacial Stresses?", Journal of Applied Physics D, vol.41, 2008

2. E. Suhir, "Stresses in Bi-Metal Thermostats", ASME Journal of Applied Mechanics, vol. 53, No. 3, Sept. 1986.

3. E. Suhir, "Predicted Mechanical Behavior of High-Tc Superconducting Ceramic Films", in T. Venkatesan, ed., "Processing of Films for High-Tc Superconducting Electronics", SPIE, Vol. 1187, 1990.

4. V. Mishkevich and E. Suhir, "Simplified Approach to the Evaluation of Thermally Induced Stresses in Bi-Material Structures", in E. Suhir, ed., "Structural Analysis in Microelectronics and Fiber Optics", ASME Press, 1993.

5. E. Suhir, "Structural Analysis in Microelectronics and Fiber Optics", Van-Nostrand, New York, 1991. 